\title{
Osteoid osteoma of the fibula in a female rugby player
}

M W J Meirhaeghe, ${ }^{1,2}$ MD, MMed (Phys Med \& Rehab); P L Viviers, ${ }^{1}$ MB ChB, MMedSc, MSc (Sports Med); J H Kirby, ${ }^{1} \mathrm{MB}$ ChB, MSc (Sports Med); J T Viljoen, ${ }^{1}$ BSc Physiotherapy, MPhil (Exerc Sci)

\author{
${ }^{I}$ Stellenbosch University, Campus Health Services and the Centre for Human Performance Sciences, Stellenbosch, South Africa \\ ${ }^{2}$ Catholic University of Leuven, Department of Physical Health and Rehabilitation, Leuven, Belgium
}

Corresponding author: P L Viviers (plv@sun.ac.za)

\begin{abstract}
Osteoid osteomas are benign osteoblastic tumours encountered relatively commonly among skeletal lesions. Despite distinct clinical findings, atypical presentations make for a challenging or delayed diagnosis which may negatively affect a patient's quality of life in the interim. We present the case of a young female rugby player with a subperiosteal osteoid osteoma of the distal fibula - a rare location for this type of tumour.
\end{abstract}

S Afr J SM 2013;25(4):114-115. DOI:10.7196/SAJSM.499

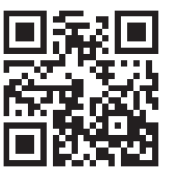

Osteoid osteomas (OOs) may account for almost $12 \%$ of all benign bone tumours. ${ }^{[1]}$ These skeletal neoplasms are most common among young patients (aged 10 - 30 years) and there is also a strong male preponderance, with a 3:1 male to female ratio. ${ }^{[2,3]}$ Classically, OO presents as an intracortical lesion of the shaft occurring in the long bones; however, its location has also proven to be subperiosteal, endosteal and even medullary, albeit less common. ${ }^{[2,4]}$ The most frequently affected sites include the femur and tibia (roughly $50 \%$ of all cases), while less regular manifestations include the humerus, spinal column and phalanges of the hands and feet. ${ }^{[2-4]}$ Morphologically, tumours are characterised by an osteoid-rich central nidus embedded in a fibrous stroma of vascular connective tissue; ${ }^{[2,5]}$ this rarely exceeds $2 \mathrm{~cm}$ in diameter and is surrounded by a zone of sclerotic bone tissue due to reactive bone formation. ${ }^{[3,6,7]}$

Patients invariably complain of pain that is most severe at night and which is usually relieved with salicylates or non-steroidal anti-inflammatory drugs (NSAIDs). ${ }^{[3]}$ Local tenderness is typically the only finding during physical examination, although warmth, swelling and erythema are also possible, albeit less common. ${ }^{[2]}$ On the whole, from a prognostic standpoint, some OO cases have been known to regress spontaneously; ${ }^{[2,8]}$ however, the majority require surgical intervention.
We report the case of a young female rugby player with a subperiosteal OO located within the distal fibula. We wish to emphasise that unusual presentations of $\mathrm{OO}$ may be misleading for clinical practitioners, and can even result in inaccurate diagnoses.

\section{Case report}

A 23-year-old female elite rugby player presented with longstanding left lateral lower-leg pain while performing high-speed running. There was no long-term improvement with rest, physiotherapy or treatment with NSAIDs. Overall, the patient's medical history was unremarkable and there was no associated nocturnal pain.
The initial physical examination showed slight tenderness upon palpation of the distal fibula as well as discomfort during resisted eversion of the foot. The patient experienced pain when performing one-leg hops as well as discomfort when a vibrating tuning fork was placed over the area. Plain radiographs showed a discrete hypodensity on the medial side of the distal fibula (Fig. 1A). After further in-depth investigation, magnetic resonance imaging (MRI) revealed oedema adjacent to a lesion in the bone (Fig. 1B). Finally, a computed tomography (CT) scan confirmed the presence of a cystic tumour with surrounding osteosclerosis located just below the periosteum (Fig. 1C).
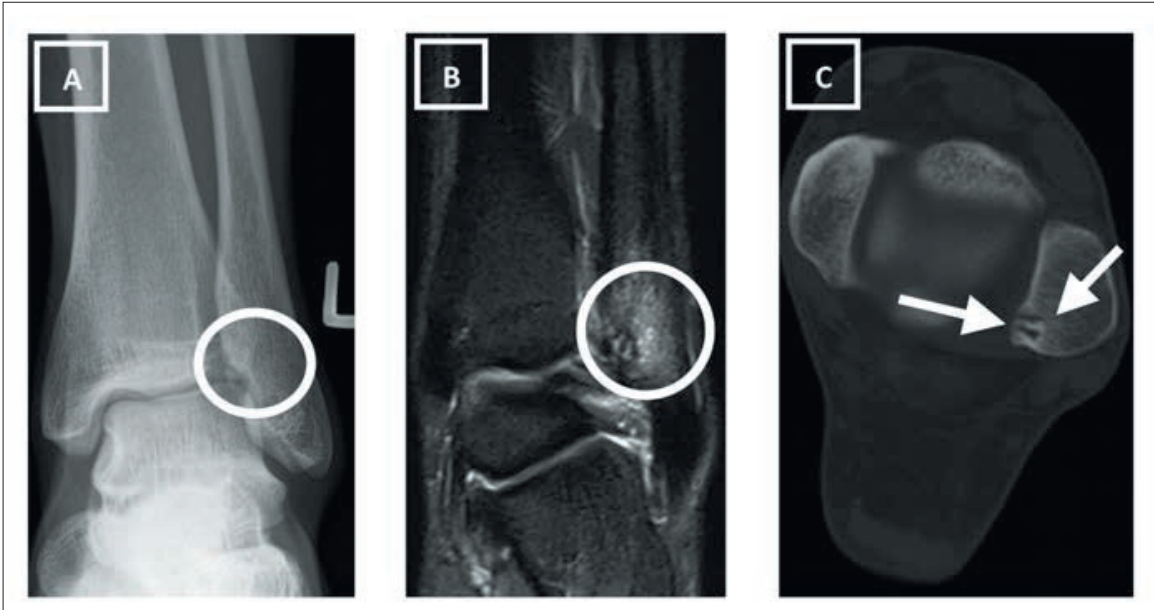

Fig. 1. (A) Anteroposterior radiograph and (B) magnetic resonance imaging of the osteoid osteoma situated within the left distal fibula. (C) A cross-sectional computed tomography scan shows the clearly demarcated subperiosteal lesion within the distal medial fibula. 
Based on these findings, the tumour was excised surgically using a posterolateral approach to the distal fibula. Following successful removal of the bone mass, further inspection revealed characteristic features of a nidus, which was confirmed histologically as being an OO. Mobilisation with partial weight-bearing for six weeks and physiotherapist-guided functional rehabilitation took place. The eventual outcome after three months was that the patient was able to run without pain and returned to play.

\section{Discussion}

Since OO can occur at a wide variety of locations and is often accompanied by a lack of radiological findings, diagnosis can be complex, and in many instances even overlooked. ${ }^{[7]}$ We encountered a similar problem, considering that the fibula is a rare localisation for an $\mathrm{OO}$ and that our patient displayed an atypical presentation at first (i.e. lack of nocturnal pain and failure to respond to NSAIDs). Subsequently, a number of different diagnoses were considered, including bony stress reaction, fibular stress fracture, as well as tendinopathy of the peroneal tendons. Less likely considerations were impingement syndrome, sinus tarsi syndrome, dislocation of the peroneal tendons and referred pain. Lastly, infections and tumours were also considered; however, the clinical presentation thereof made these options less likely.

Plain radiography is usually the most cost-effective examination and is often also all that is required to ensure the accurate diagnosis of OO. Nonetheless, it should be noted that during the first few months after the onset of pain, initial radiographs often appear to be normal, which can be misleading. As a result, it is useful to repeat a radiograph if an $\mathrm{OO}$ is suspected. We found this to be valid, considering that we initially took a plain radiograph that was reported as normal. However, retrospectively, a discrete hypodensity could be observed. Furthermore, the use of detailed imaging techniques such as CT and MRI is invaluable when it comes to precise localisation of the tumour and in making a definitive diagnosis.

First-line medical treatment for this syndrome essentially consists of proper pain management through the use of aspirin or NSAIDs. Still, many patients are unable to continue with this type of treatment due to uncontrolled pain or contraindications to NSAID use. There are also several available surgical treatment options for effective excision of the tumour. Traditionally, the treatment of choice has been open surgery with unroofing and curettage; however, a number of newer, less invasive techniques such as CT-guided percutaneous treatment are also being used and can have a primary cure rate of $100 \%{ }^{[2,8]}$ Regardless of the method of treatment, exact localisation of the lesion, before and during the procedure, is a very important determinant of success.

\section{Conclusion}

The subperiosteal region of the distal fibula is an uncommon location for an OO. Clinical presentation and radiological findings can be misleading, which often results in a delay in diagnosis. In some instances, this phenomenon may even masquerade as an alternative form of pathology or injury. Ultimately, delayed diagnosis or misinterpretation of clinical signs and symptoms have the potential to affect early clinical decision-making processes governing optimal patient management. As a result, OO should always be considered when pain persists, even if initial X-rays appear to be normal. The goal of treatment is rapid and enduring pain relief, but also a safe return to sport in athletic populations.

Acknowledgements. Dr RVP de Villiers from Van Wageningen and Partners radiology practice for the images.

\section{References}

1. Dahlin DC, Unni KK. Bone Tumors: General Aspects and Data on 8542 Cases. 4th ed. Thomas, Springfield, 1987:88-101.

2. Lee EH, Shafi M, Hui JHP. Osteoid osteoma: A current review. J Pediatr Orthop 2006;26:695-700.

3. Greenspan A. Benign bone-forming lesions: Osteoma, osteoid osteoma and osteoblastoma. Clinical, imaging, pathologic and differential considerations. Skeletal Radiol 1993;22(7):485-500

4. Kayser F, Resnick D, Haghighi P, et al. Evidence of the subperiosteal origin of osteoid osteomas in tubular bones: Analysis by CT and MR imaging. Am J Roentgenol 1998;170:609-614.

5. Chronopoulos E, Xypnitos FN, Nikolaou VS, et al. Osteoid osteoma of a metacarpal bone: A case report and review of the literature. J Med Case Rep 2008;2:285-288.

6. Jaffe HL. Osteoid osteoma of bone. Radiology 1945;45:319.

7. Chai JW, Hong SH, Choi JY, et al. Radiologic diagnosis of osteoid osteoma: From simple to challenging findings. Radiographics 2010;30(3):737-749.

8. Cantwell CP, Obyrne J, Eustace S. Current trends in treatment of osteoid osteoma with an emphasis on radiofrequency ablation. Eur Radiol 2004;4(4):607-617. 